\title{
Genetic evaluation of the effect of GLCCI1 (a) CrossMark rs37973 on corticosteroid response in chronic obstructive pulmonary disease
}

Michael Mosteller ${ }^{1 \dagger}$, Maarten van den Berge ${ }^{2 \dagger}$, Louise Hosking ${ }^{3}$, Wim Timens ${ }^{4}$, Pieter S. Hiemstra ${ }^{5}$, Courtney Crim ${ }^{6}$, Dirkje S. Postma ${ }^{2+}$ and Soumitra Ghosh ${ }^{7^{*+}}$

\begin{abstract}
Background: The efficacy of inhaled corticosteroids (ICS) for chronic obstructive pulmonary disease (COPD) varies between patients, which may be partially due to genetic differences. A single-nucleotide polymorphism, rs37972, in the glucocorticoid-induced transcript 1 gene (GLCCI1) has been associated with variations in response (forced expiratory volume in $1 \mathrm{~s}\left[\mathrm{FEV}_{1}\right]$ and residual volume) to fluticasone propionate (Groningen and Leiden Universities Study of Corticosteroids in Obstructive Lung Disease [GLUCOLD] study). The aim of this study was to determine whether variation in the GLCCI1 gene at rs37973 is associated with ICS response in patients with COPD.
\end{abstract}

Methods: Variations in the GLCCI1 gene, rs37973 (which is in almost complete linkage disequilibrium with rs37972) were examined in 402 corticosteroid-treated, non-Hispanic Caucasian COPD patients, and in 63 GLUCOLD study patients.

Results: We were unable to confirm a genetic association between GLCCI1 and change in $\mathrm{FEV}_{1}$, unlike equivalent data generated for rs37973 from the GLUCOLD study sample. This was despite accounting for differences in gender, baseline $\mathrm{FEV}_{1}$, severity of COPD, extent of reversibility, and combination therapy of ICS with bronchodilators.

Conclusions: We conclude that based on changes in $\mathrm{FEV}_{1}$, there is no evidence that the GLCCI1 variant rs37973 has an impact on corticosteroid response in patients with COPD.

Trial registration: GSK study number HZC112206 (ClinicalTrials.gov identifier: NCT01053988. Registered January 14 2010) and HZC112207 (ClinicalTrials.gov identifier: NCT01054885. Registered January 14 2010).

Keywords: Chronic obstructive pulmonary disease, Inhaled corticosteroids, Genetic association, GLCCl1, Fluticasone furoate, Fluticasone propionate

\section{Background}

Worldwide, more than 300 million people suffer from chronic obstructive pulmonary disease (COPD), a progressive respiratory disease for which there is no cure. Treatments such as long-acting beta-agonists and longacting anticholinergic drugs with and without inhaled corticosteroids (ICS), may improve quality of life, and reduce symptoms and the frequency of COPD exacerbations [1].

The response to treatment for COPD may be differentially influenced by the nature of the underlying disease

\footnotetext{
* Correspondence: soumitra.x.ghosh@gsk.com

${ }^{\dagger}$ Equal contributors

${ }^{7}$ GlaxoSmithKline, 709 Swedeland Road, UW2230, King of Prussia, PA

19406-0939, USA

Full list of author information is available at the end of the article
}

and the genetic background of the patient. Previous reports have suggested that the minor allele of the glucocorticoid-induced transcript 1 gene (GLCCI1) promoter variant rs37972 is associated with a poorer ICS treatment response in Caucasians with asthma [2]. GLCCl1 encodes the glucocorticoid-induced transcript 1 gene, which has been suggested to be an early marker of glucocorticoid-induced apoptosis [3]. The study by Tantisira et al. [2] also showed that the minor allele of rs37973, which is in almost complete linkage disequilibrium with $\operatorname{rs} 37972\left(r^{2}=0.97\right)$, was associated with reduced expression of GLCCI1. A further study found no statistically significant association between the GLCCI1 single-nucleotide polymorphism (SNP) rs37973 and ICS responsiveness in patients with asthma [4]; 
however, the direction of the effect was the same as reported in the study by Tantisira et al. [2]. Vijverberg et al. also found that the GLCCI1 gene had no effect on ICS response in children with asthma [5]. In a study of Japanese patients with asthma, those who were homozygous for the rs37973 minor allele (GG), experienced a greater decline in lung function over a 4-year period than patients with other rs37973 genotypes independently of ICS use [6]. Taken together, these studies suggest that compared with the major allele, the minor allele of the GLCCI1 SNP rs37973 could have a poorer response to ICS treatment in patients with asthma, though with a small effect size.

Previously, van den Berge et al. [7] reported that patients with moderate-to-severe COPD who carried the minor allele $(\mathrm{T})$ of GLCCI1 SNP rs37972 had a poorer lung function response to ICS treatment than patients who were homozygous for the major allele (CC) [6]. Although the study only assessed 63 patients, these results may be important, as they may help to develop strategies for personalized treatment in patients with COPD.

The present study (GSK study number 200367) evaluated the potential association between GLCCI1 rs37973 and ICS response as a post-hoc analysis using a much larger sample from two double-blind, randomized trials of the ICS, fluticasone furoate (FF), in patients with moderate-to-severe COPD. The aim of this study was to determine whether variation in the GLCCI1 gene at rs37973 is associated with the FEV1 response to ICS in patients with COPD.

\section{Methods}

\section{Patients}

Patients were taking part in one of two 6-month doubleblind, randomized, controlled trials, from which the primary results have been published previously HZC112206 (Study 1: Kerwin et al. [8], ClinicalTrials.gov identifier: NCT01053988) and HZC112207 (Study 2: Martinez et al. [9], ClinicalTrials.gov identifier: NCT01054885), which evaluated the efficacy and safety of FF in patients with COPD. Study participants included in this analysis consented to genetic research, provided a DNA sample, were randomized to receive FF monotherapy only, and self-reported as non-Hispanic whites. Among the 402 Caucasian patients who satisfied these criteria, 148 (36.8\%) were treated with FF inhalation powder $100 \mu \mathrm{g}$ once daily (OD) and the remainder with FF $200 \mu \mathrm{g}$ OD; for these analyses, treatment effect was assessed at 3 months. All patients were adults of $\geq 40$ years of age.

All patients with COPD participating in the Groningen and Leiden Universities Study of Corticosteroids in Obstructive Lung Disease (GLUCOLD) study [10] were also included in this analysis. In this double-blind study, patients were randomly assigned to receive one of four treatments: 1) fluticasone propionate (FP) $500 \mu \mathrm{g}$ twice daily (BID) for 30 months; 2) $\mathrm{FP} /$ salmeterol 500/50 $\mu \mathrm{g}$ BID for 30 months; 3) placebo BID for 30 months; or 4) FP $500 \mu \mathrm{g}$ BID for the first 6 months followed by placebo BID for 24 months.

All studies were performed in accordance with the Declaration of Helsinki and approved by local medical ethics committees and all patients gave their written informed consent to participate.

\section{Genetic markers}

Genotyping for the rs37972 and rs37973 SNPs was performed for Studies 1 and 2, and the GLUCOLD study by competitive allele-specific polymerase chain reaction amplification of target sequences and endpoint fluorescence genotyping (KASPar ${ }^{\mathrm{rm}}$ ) KBiosciences (Hoddesdon, UK). Here, we report on rs37973 only, as this variant is known to alter GLCCI1 gene expression and is in almost complete linkage disequilibrium $\left(r^{2}=0.97\right)$ with $\mathrm{rs} 37972$ [2].

\section{Primary endpoints}

For these analyses, the primary endpoint was the strength of association between the rs37973 SNP and change in the forced expiratory volume in $1 \mathrm{~s}\left(\mathrm{FEV}_{1}\right)$ at Week 12, reported as percent of predicted $\mathrm{FEV}_{1}$ ( $\mathrm{FEV}_{1}$ \%pred), adjusting for variables nominally associated with change of $\mathrm{FEV}_{1}$ \%pred at Week 12. The GLUCOLD study had the same primary endpoint.

Clinical response was defined as change in trough (pre-bronchodilator) $\mathrm{FEV}_{1}$ \%pred from baseline to Day 84 (Week 12) of treatment with FF. For Studies 1 and 2, trough $\mathrm{FEV}_{1}$ was calculated by taking the mean of the two $\mathrm{FEV}_{1}$ measurements at 23 and $24 \mathrm{~h}$ following the Day 83 dose. In the GLUCOLD study, we measured change in post-bronchodilator $\mathrm{FEV}_{1}$ also after 12 weeks.

\section{Statistical methods}

In Studies 1 and 2, a sample size of 402 gave statistical power of approximately $100 \%$ to demonstrate an additive association between rs37973 and change in $\mathrm{FEV}_{1}$ \%pred with ICS treatment. This calculation assumed a 1-sided test, a minor allele frequency of $40 \%$, an alpha error of 0.05 , a mean change between the homozygotes of $5.4 \%$, and genotype-specific standard errors of $\pm 4.9 \%$ and $\pm 6.9 \%$ for CC and TT, respectively, as previously published [7].

Preliminary analyses indicated that there were statistically significant differences in patient characteristics (baseline $\mathrm{FEV}_{1}-200 \mathrm{ml}$ ) between those participating in Study 1 and those participating in Study 2. Therefore, associations between genotype and changes in $\mathrm{FEV}_{1}$ \%pred with ICS treatment were assessed separately within each study and the results combined using an inverse variance-weighted meta-analysis that assumed fixed effects. 
For Study 1, the distribution of the change in $\mathrm{FEV}_{1}$ \%pred was approximately normal and no data transformation was necessary. For Study 2, the Shapiro-Wilk statistic was highly significant; therefore, an inverse normal transformation was applied.

Covariate selection was conducted separately for Studies 1, 2, and the GLUCOLD study. The covariates investigated included age, gender, height, weight, body mass index (BMI), country, study center group, smoking status (current or former), pack-years smoking, $\mathrm{FEV}_{1}$ at baseline, $\mathrm{FEV}_{1} \%$ pred at baseline, forced vital capacity (FVC), and $\mathrm{FEV}_{1} / \mathrm{FVC}$ at baseline. After identifying variables that had univariate significant associations with the response variable, a forward-reverse stepwise analysis was performed in which the criterion for entry into the model was $p=0.25$ and the criterion for staying in the model was $p=0.05$. After following this procedure, only pack-years smoking was retained for Study 1, and only $\mathrm{FEV}_{1} \%$ pred at baseline was retained for Study 2. In the GLUCOLD study, BMI and smoking status were retained in the final model.

Linear regression with an additive model was used to assess the effect of rs37973 on change from baseline in trough $\mathrm{FEV}_{1}$ \%pred from baseline. This was performed separately for Studies 1 and 2. The statistical model included the rs37973 genotype, coded as 0,1 , or 2 based on the number of minor alleles present, and the covariates identified by the covariate selection process within each study.

\section{Results}

The clinical characteristics of patients included in Study 1,2 , and the GLUCOLD study are presented in Table 1. The main difference between the studies (Table 1; Additional file 1: Tables S1 and S2) was that patients in Studies 1 and 2 had more severe COPD at baseline than patients in the GLUCOLD study.

In Studies 1 and 2, the frequency of the rs37973 minor allele (G) was $42.5 \%$, comparable with earlier publications $[2,4,6]$. There was no evidence for deviation from Hardy-Weinberg equilibrium $(p=0.54)$.
The frequencies for rs37973 genotypes AA, AG, and GG in Study $1(n=94)$ were $28 \%, 54 \%$, and $18 \%$, respectively, and in Study $2(n=308)$ were $36 \%, 45 \%$, and $19 \%$, respectively. For the GLUCOLD study $(n=63)$, the frequencies were $33 \%, 50 \%$, and $17 \%$, respectively.

In Study $1(n=94)$, there was no evidence for an association between the minor allele and poorer response based on change in trough $\mathrm{FEV}_{1} \%$ pred (1-sided $p=0.17$ ). Analysis of unadjusted change in $\mathrm{FEV}_{1}$ \%pred by rs37973 genotype (Fig. 1a) indicates that the genotype with the numerically poorest response was AG ( $p=0.036$ for AA versus AG; $p=0.16$ for GG versus AG). This is in contrast to previous findings where patients with the TT genotype (rs37972) had the poorest response [6].

In Study $2(n=308)$, there was no association between rs37973 genotypes and change in trough $\mathrm{FEV}_{1} \%$ pred (unadjusted values, Fig. 1b; 1 -sided $p=0.98$ ). A sensitivity analysis exploring the effect of transformation and covariate adjustment on change in $\mathrm{FEV}_{1}$ by rs37973 genotype was performed in Study 2 using four different scenarios; in general, the results were similar in each scenario (Additional file 1: Appendix 1; Table S3).

In the GLUCOLD study $(n=63)$, the heterozygote (AG) and minor allele homozygote (GG) genotypes were associated with less improvement in $\mathrm{FEV}_{1} \%$ pred compared with the major allele homozygotes (AA; $p=0.045$ and $p=0.048$, respectively; Fig. 1c).

\section{Meta-analysis of study 1 and 2}

The combined estimate for the additive effect of the rs37973 minor allele on change in trough $\mathrm{FEV}_{1} \%$ pred (adjusted for covariates) was significantly different from 0 at $0.16 \%$ (2-sided $p=0.037 ; 95 \%$ confidence interval: $0.01-0.31$ ). However, in contrast with previous observations [7], the parameter estimate was positive, indicating that the minor allele (G) of rs37973 was associated with a better $\mathrm{FEV}_{1}$ \%pred response (Fig. 1b) versus the major allele rather than the expected poorer response. A 1sided test of the null hypothesis that the minor allele is associated with a better response to FF treatment was not rejected at $p=0.98$.

Table 1 Comparison of baseline characteristics and change in trough FEV 1 at week 12 in studies 1 and 2, and in the GLUCOLD study

\begin{tabular}{|c|c|c|c|c|}
\hline Variables & Study $1(n=94)$ & Study $2(n=308)$ & $p$ value & GLUCOLD $(n=63)$ \\
\hline Age, years & $61.3(0.9)$ & $61.2(0.5)$ & 0.937 & $62.1(1.02)$ \\
\hline $\mathrm{BMI}, \mathrm{kg} / \mathrm{m}^{2}$ & $27.5(0.6)$ & $27.1(0.3)$ & 0.482 & $25.5(0.48)$ \\
\hline $\mathrm{FEV}_{1}$ at baseline, $\mathrm{L}$ & $1.2(0.04)$ & $1.4(0.03)$ & 0.008 & $2.0(0.05)$ \\
\hline FEV $1 \%$ pred at baseline & $42.1(1.1)$ & $44.5(0.8)$ & 0.113 & $63.1(1.1)$ \\
\hline $\mathrm{FEV}_{1} / \mathrm{FVC}$ at baseline, $\%$ & $48.3(1.2)$ & $46.1(0.7)$ & 0.111 & $50.8(1.1)$ \\
\hline Change in trough $\mathrm{FEV}_{1}$ as a \% of predicted at Week 12 & $2.5(0.8)$ & $0.5(0.4)$ & 0.013 & $0.5(0.8)$ \\
\hline
\end{tabular}

$p$ values shown are for differences between Studies 1 and 2

Values are mean (standard error) unless otherwise stated

$B M I$, body mass index, $F E V_{1}$, forced expiratory volume in $1 \mathrm{~s}, F E V_{1} \%$ pred percent of predicted forced expiratory volume in $1 \mathrm{~s}, F V C$ forced vital capacity, GLUCOLD Groningen and Leiden Universities Study of Corticosteroids in Obstructive Lung Disease 

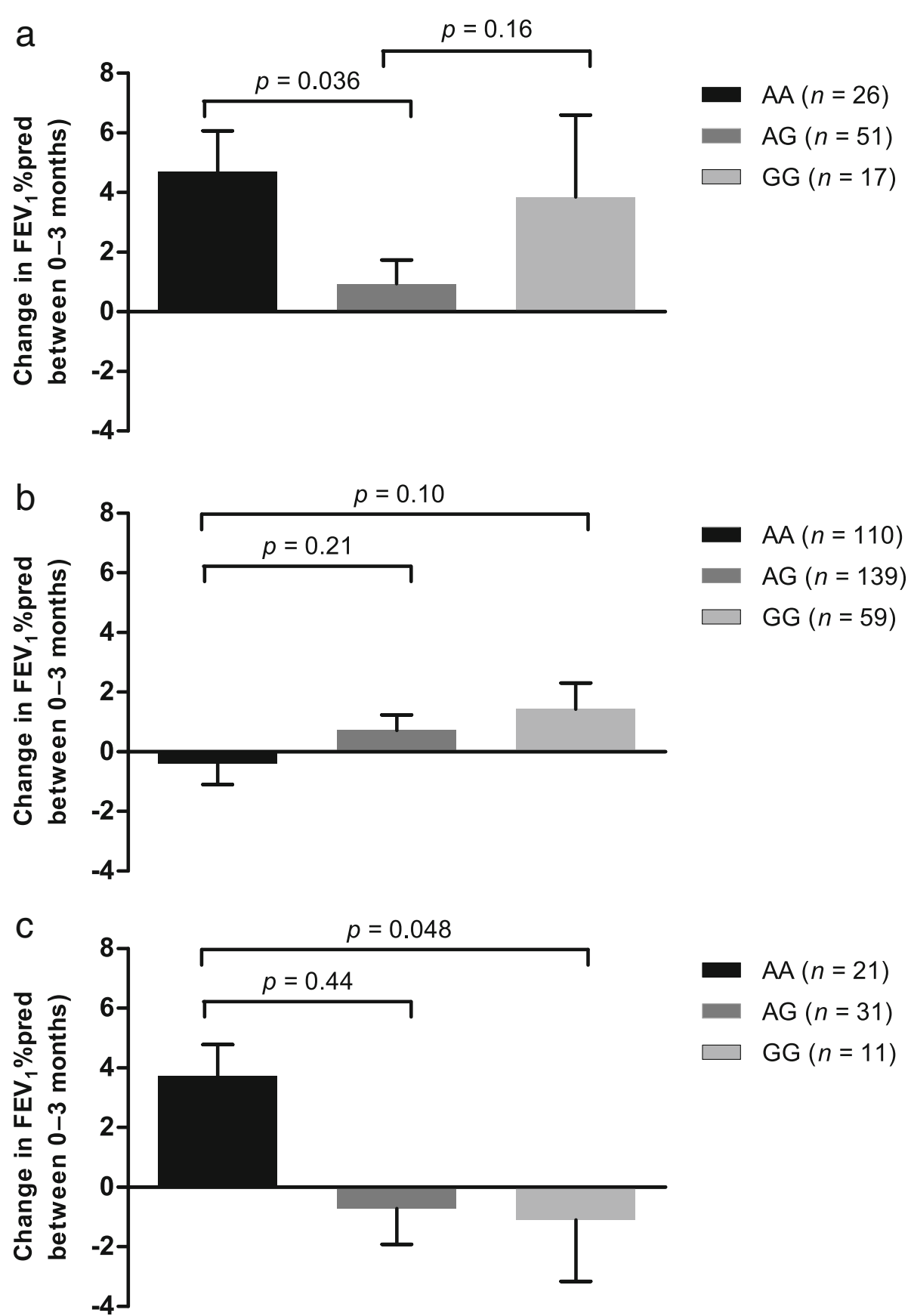

Fig. 1 a Unadjusted change in $\mathrm{FEV}_{1} \%$ pred in Study 1 by rs 37973 genotype; b unadjusted change in $\mathrm{FEV}_{1} \%$ pred in Study 2 by rs 37973 genotype; c unadjusted change in $\mathrm{FEV}_{1} \%$ pred in the GLUCOLD study by rs 37973 genotype $\mathrm{FEV}_{1} \%$ pred, percent of predicted forced expiratory volume in 1 s; GLUCOLD, Groningen and Leiden Universities Study of Corticosteroids in Obstructive Lung Disease

\section{Discussion}

COPD is a heterogeneous disease that shows some inter-individual variability in response to several drugs. Compared with COPD patients carrying the major allele (C) of the GLCCI1 SNP rs37972, patients carrying the minor allele $(\mathrm{T})$ have been previously shown to have a poorer 3-month and 6-month $\mathrm{FEV}_{1}$ response to ICS treatment [7]. To assess the strength of this association, we analyzed data from two double-blind, randomized, controlled studies of non-Hispanic Caucasian patients treated with FF. We found no significant association between the minor allele of the functional GLCCI1 SNP rs37973 (G) and poorer ICS treatment response in either of the two independent COPD cohorts studied. In contrast to previous studies, a meta-analysis of the two independent COPD cohorts (Studies 1 and 2) showed that 
the rs37973 minor allele (G) was associated with a statistically significant improvement in $\mathrm{FEV}_{1}$ \%pred after 3 months of FF treatment.

The results from the GLUCOLD study presented in this paper are very similar, but not identical to, the original publication [7]. In the current study, we genotyped samples for the functional SNP, rs37973, that is highly correlated with rs37972. To compare directly with the results of the two independent COPD cohorts (Studies 1 and 2) we performed a linear regression under an additive model with covariates. In contrast, data in the original publication [7] were analysed using an unpaired $t$ test. These features may explain the slight discrepancy in $p$-values between the two studies.

We considered several possible factors that might explain the contradictory findings in the GLUCOLD study compared with the two independent COPD cohorts. Firstly, it is important to mention that in the GLUCOLD study, patients with COPD who received FP monotherapy or combined FP and salmeterol were analyzed together, whereas the analyses of Studies 1 and 2 were performed in COPD patients treated with FF alone. For this reason, we re-analyzed our data from the GLUCOLD study patients treated with FP alone, and similarly found that the minor allele $(G)$ still predicted smaller changes in $\mathrm{FEV}_{1}$ after 3 months of treatment, thus not explaining differences in results.

Secondly, the GLUCOLD study predominantly included males, whereas the Study 1 and 2 cohorts included both males and females. However, a gender-specific separate analysis of males and females gave results that were comparable to the total GLUCOLD study sample.

We also investigated subsets of Studies 1 and 2 to align our analysis with the features of the GLUCOLD study [7]. Previous ICS use, including run-in time, did not explain the differences in ICS response by genotype between Studies 1 and 2 and the GLUCOLD studies. Differences between studies in baseline $\mathrm{FEV}_{1}$, severity of disease, and the extent of reversibility did not explain the differences observed in response by genotype. Further, the GLUCOLD study differed in dosage and duration of treatments compared with Studies 1 and 2. However, we found no dosage effect and we were only able to compare treatment outcome after 3 months for the three studies.

Finally, the GLUCOLD study examined additional pulmonary function parameters, such as residual volume (RV). We were unable to replicate these results as the information on RV was unavailable in Studies 1 and 2, and unfortunately, it has no clear surrogate.

\section{Conclusions}

We conclude that the minor allele of the GLCCI1 SNP rs37973 (G) is not associated with poorer ICS responsiveness in COPD, as measured by change in trough
$\mathrm{FEV}_{1}$ \%pred. In contrast, in some patients, it may even predict a better outcome with ICS treatment. We thus did not replicate earlier findings in the GLUCOLD study. Significant disease heterogeneity makes it difficult to compare data from the three studies. However, we have excluded the major factors that led to this heterogeneity as reasons for our inability to confirm the findings from the original study.

\section{Additional file}

Additional file 1: Table S1. Clinical characteristics of patients with COPD by rs37973 genotype (Studies 1 and 2). Table S2. Clinical characteristics of patients with COPD by rs37973 genotype (GLUCOLD study). Appendix 1. Sensitivity analyses exploring the effect of transformation and covariate adjustment in Study 2. Table S3. The influence of transformation and covariate adjustment on the analysis of change in $\mathrm{FEV}_{1}$ versus rs37973 genotype in patients with COPD (Study 2). Appendix 2. List of Institutional Review Boards/Independent Ethics Committees and the Chairperson (s) for each site. (DOCX $68 \mathrm{~kb}$ )

\section{Abbreviations}

BID: Twice daily; BMI: Body mass index; COPD: Chronic obstructive pulmonary disease; FEV $\%$ pred: Percent of predicted forced expiratory volume in $1 \mathrm{~s}$; $\mathrm{FEV}_{1}$ : Forced expiratory volume in $1 \mathrm{~s}$; FF: Fluticasone furoate; FP: Fluticasone propionate; FVC: Forced vital capacity; GLCCI1: Glucocorticoid-induced transcript 1 gene; GLUCOLD: Groningen and Leiden Universities study of corticosteroids in obstructive lung disease; ICS: Inhaled corticosteroids; OD: Once daily; RV: Residual volume; SNP: Single-nucleotide polymorphism

\section{Acknowledgments}

We would like to thank Kay Murphy for leading the efforts on genotyping the samples for Study 1 and 2. Editorial support in the form of editorial suggestions to draft versions of this paper, assembling tables and figures, and collating author comments was provided by Angela Rogers PhD, at Gardiner-Caldwell Communications (Macclesfield, UK) and was funded by GlaxoSmithKline.

\section{Funding}

This study was funded by GlaxoSmithKline (Study number 200367). GSK were involved in the design of the study, data collection, analysis, and interpretation and in writing the manuscript (the following authors are GSK employees: MM, LH, CC, SG). Medical writing services employed in development of the manuscript were funded by GSK.

\section{Availability of data and materials}

The datasets supporting the conclusions of this article are included within the article (and its additional files). A data summary is available here: http:// www.gsk-clinicalstudyregister.com/study/200367\#rs.

\section{Authors' contribution}

All authors contributed to the conception and design of the study and to data interpretation. MM, MvdB, DP, SG, WT, and PH contributed to data acquisition. MM, MvdB, DP, and SG contributed to the data analysis, interpretation, and writing of the manuscript. All authors commented on and approved the final version of the manuscript.

\section{Competing interests}

MM declares being an employee of GlaxoSmithKline (GSK) and owning stocks and shares in GSK. MvdB has received research grants (paid to the University) from GSK, Chiesi, and TEVA. LH and SG are currently employees of GSK and both hold shares in GSK. WT has received personal fees from Pfizer, GSK, Chiesi, Roche Diagnostics/Ventana, Biotest, Merck Sharp \& Dohme, Novartis, and Lilly Oncology. He has also received grants from the Dutch Asthma Fund. PSH has received grants from The Netherlands Organization for Scientific Research, The Netherlands Asthma Foundation, and GSK. CC is currently an employee of GSK. The University of Gröningen received grant 
funding for DSP to carry out research from Astra Zeneca, Chiesi, Genentec, GSK, and Roche. Additionally, fees for consultancies (paid to the University) have been received from Astra Zeneca, Boehringer Ingelheim, Chiesi, GSK, Takeda, and TEVA.

\section{Consent for publication}

Not applicable.

\section{Ethics approval and consent to participate}

All studies were performed in accordance with the Declaration of Helsinki and approved by local medical ethics committees (Additional file 1: Appendix 2) and all patients gave their written informed consent to participate.

\section{Author details}

${ }^{1}$ Parexel International, Durham, NC, USA. ${ }^{2}$ Department of Pulmonary Diseases, University of Groningen, University Medical Center Groningen, Groningen, The Netherlands. ${ }^{3}$ GlaxoSmithKline, Stevenage, UK. ${ }^{4}$ Department of Pathology and Medical Biology, University of Groningen, University Medical Center Groningen, Groningen, The Netherlands. ${ }^{5}$ Department of Pulmonology, Leiden University Medical Center, Leiden, The Netherlands. ${ }^{6}$ GlaxoSmithKline, Research Triangle Park, Durham, NC, USA.

'GlaxoSmithKline, 709 Swedeland Road, UW2230, King of Prussia, PA 19406-0939, USA.

Received: 9 August 2016 Accepted: 2 January 2017

Published online: 17 January 2017

\section{References}

1. Tashkin DP, Ferguson GT. Combination bronchodilator therapy in the management of chronic obstructive pulmonary disease. Respir Res. 2013;14:49.

2. Tantisira KG, Lasky-Su J, Harada M, Murphy A, Litonjua AA, Himes BE, et al. Genomewide association between GLCCI1 and response to glucocorticoid therapy in asthma. N Engl J Med. 2011;365:1173-83.

3. Chapman MS, Askew DJ, Kuscuoglu U, Miesfeld RL. Transcriptional control of steroid-regulated apoptosis in murine thymoma cells. Mol Endocrinol. 1996;10:967-78.

4. Hosking L, Bleecker E, Ghosh S, Yeo A, Jacques L, Mosteller M, et al. GLCCI1 rs37973 does not influence treatment response to inhaled corticosteroids in white subjects with asthma. J Allergy Clin Immunol. 2014;133:587-9.

5. Vijverberg SJ, Tavendale R, Leusink M, Koenderman L, Raaijmakers JA, et al. Pharmacogenetic analysis of GLCCI1 in three north European pediatric asthma populations with a reported use of inhaled corticosteroids. Pharmacogenomics. 2014;6:799-806.

6. Izuhara $Y$, Matsumoto $H$, Kanemitsu $Y$, Izuhara $K$, Tohda $Y$, Horiguchi T, et al. GLCCI1 variant accelerates pulmonary function decline in patients with asthma receiving inhaled corticosteroids. Allergy. 2014;69:668-73.

7. van den Berge M, Hiemstra PS, Postma DS. Genetics of glucocorticoids in asthma. N Engl J Med. 2011;365:2434-5.

8. Kerwin EM, Scott-Wilson C, Sanford L, Rennard S, Agusti A, Barnes N, et al. A randomized trial of fluticasone furoate/vilanterol $(50 / 25 \mu \mathrm{g} ; 100 / 25 \mu \mathrm{g})$ on lung function in COPD. Respir Med. 2013;107:560-9.

9. Martinez FJ, Boscia J, Feldman G, Scott-Wilson C, Kilbride S, Fabbri L, et al. Fluticasone furoate/vilanterol $(100 / 25 ; 200 / 25 \mu \mathrm{g})$ improves lung function in COPD: a randomized trial. Respir Med. 2013;107:550-9.

10. Lapperre TS, Snoeck-Stroband JB, Gosman MM, Jansen DF, van Schadewijk A, Thiadens HA, et al. Effect of fluticasone with and without salmeterol on pulmonary outcomes in chronic obstructive pulmonary disease: a randomized trial. Ann Intern Med. 2009;151:517-27.

\section{Submit your next manuscript to BioMed Central and we will help you at every step:}

- We accept pre-submission inquiries

- Our selector tool helps you to find the most relevant journal

- We provide round the clock customer support

- Convenient online submission

- Thorough peer review

- Inclusion in PubMed and all major indexing services

- Maximum visibility for your research

Submit your manuscript at www.biomedcentral.com/submit
C Biomed Central 